\title{
Optical Coherence Tomography in Archaeological and Conservation Science - A new emerging field
}

\author{
Haida Liang ${ }^{* a}$, Borislava Peric ${ }^{a}$, Michael Hughes ${ }^{b}$, Adrian Podoleanu ${ }^{b}$, Marika Spring ${ }^{c}$, Stefan \\ Roehrs ${ }^{\mathrm{d}}$ \\ ${ }^{a}$ School of Science and Technology, Nottingham Trent University, Nottingham NG11 8NS, UK; \\ ${ }^{\mathrm{b}}$ School of Physical Sciences, University of Kent, Canterbury, CT2 7NR, UK; \\ 'Scientific Department, The National Gallery, London WC2N 5DN, UK \\ ${ }^{\mathrm{d}}$ Department of Conservation and Scientific Research, The British Museum, \\ London WC1B 3DG, UK
}

\begin{abstract}
There has been a long tradition of applying biomedical imaging techniques to the examination of historical artefacts, owing to similar demands for non-invasive methods in both fields. Optical Coherence Tomography (OCT) is no exception. We review the achievements on OCT applications to art conservation and archaeology since the publication of the first papers in 2004. Historical artefacts include a much broader range of materials than biological tissues, hence presenting a greater and somewhat different challenge to the field of OCT. New results will be presented to illustrate the various applications of OCT including both qualitative and quantitative analysis.
\end{abstract}

Keywords: optical coherence tomography, low coherence interferometry, infrared imaging, 3D imaging, refractive index, scattering, absorption, art conservation, archaeology

\section{INTRODUCTION}

There has been a long tradition of applying biomedical imaging techniques to the examination of historical artefacts owing to similar demands for non-invasive methods in both fields. For example, the introduction of X-ray imaging to the examination of paintings started soon after the invention of X-rays and its application to medicine [1]. It is then not surprising that OCT has recently been applied to paintings and other cultural artefacts. In 2004, OCT was first applied to the examination of jade [2], ceramics [3] and paintings [3,4]. Direct comparisons between OCT cross-section images and real cross-sections of paint samples showed the extent OCT cross-section imaging is able to reflect the real paint layers [6,7]. Apart from the non-invasive examination of the stratigraphy of paint and varnish layers, OCT has also been shown to be the most sensitive technique for revealing preparatory sketches or underdrawings beneath paint layers owing to its high dynamic range and depth selection capabilities [5,12]. It was shown that the high sensitivity of OCT enables the interface between different varnish layers on a painting to be seen [5]. OCT has been used for dynamic monitoring of the wetting and drying of different varnish, the examination of the difference between the roughness of the dried surface for the different types of varnish $[8,15]$ and the monitoring of the aging and solvent cleaning of varnish [9]. Measurements of refractive indices of varnish and paint samples using OCT were given in [8,9]. To exploit the fast imaging capability of Fourier-domain OCT, it has been demonstrated that a fast imaging OCT is capable of monitoring real time laser ablation of varnish layers [10] and real time tracking of canvas deformation due to environmental changes such as humidity [11]. OCT imaging of gold punch marks on paintings to identify the tools used has been demonstrated in [12]. OCT has found application in the examination of ancient glass [9,14], parchment [13] and faience [15].

In this paper, we review the different OCT applications in archaeological and conservation science. The example images shown in the paper are obtained with a Thorlabs SROCT. The SROCT is a portable Fourier-domain OCT operating at a wavelength of $930 \mathrm{~nm}$ with an axial resolution of $\sim 6 \mu \mathrm{m}$, transverse resolution of $\sim 9 \mu \mathrm{m}$ and depth range of $1.6 \mathrm{~mm}$. We

"haida.liang@ntu.ac.uk; phone 44115848 8056; fax 44115848 6636;

http://www.ntu.ac.uk/research/school_research/sat/staff/55616gp.html

1st Canterbury Workshop on Optical Coherence Tomography and Adaptive Optics

edited by Adrian Podoleanu, Proc. of SPIE Vol. 7139, 713915 - @ 2008 SPIE

CCC code: 0277-786X/08/\$18 - doi: 10.1117/12.819499

Proc. of SPIE Vol. 7139 713915-1 
have adapted the instrument for in situ examination of paintings by mounting the light weight probe on a computer controlled XYZ micrometer stage which makes it capable of scanning an area as large as $15 \mathrm{~cm} \times 15 \mathrm{~cm}$. The instrument is small and portable, operating at a safe distance of around $1 \mathrm{~cm}$ from the paint surface. The experience gained in using the instrument has helped inform the design of an en face time domain OCT for in situ application in museums which complements the capabilities of the SROCT. Section 2 is devoted to a review of OCT imaging of paintings including new results; Section 3 shows new results on OCT imaging of various other historical artefacts from the British Museum collection; Section 4 discusses the specific requirements in the specification of an OCT in the applications to paintings and other historical artefacts.

\section{OCT IMAGING OF PAINTINGS}

Western European paintings were usually painted on either panel or canvas which were normally prepared with a ground layer (e.g. chalk in animal skin glue). Depending on the style and the historical period, preparatory sketches were sometimes made before paint layers were applied. To enhance the appearance of the painting and for protection, a varnish layer would then be applied once the paint was dry. Paint consists of pigments mixed in a binding medium which in most cases are either egg tempera or oil. Most types of paint are highly scattering compared to the average biological tissue. In most paint layers, multiple scattering dominates.

\subsection{Non-invasive examination of varnish and paint layers}

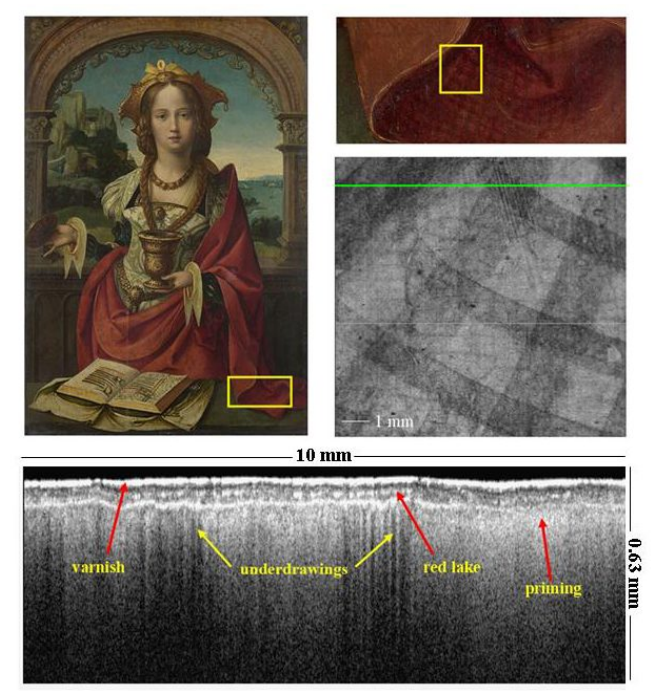

Fig. 1. OCT used to examine the layer structure of varnish and paint, revealing underdrawings of a painting. Top right: A region on the red drapery in the painting The Magdalen by an anonymous Netherlandish artist (National Gallery No. 719); Right middle: OCT en-face image at the depth of the underdrawing corresponding to the region marked by a yellow box in the top right image; Bottom: OCT cross-section image of the region marked by a green line segment on the en-face underdrawing image.

Figure 1 shows an OCT cross-section image of a painting where a varnish layer, a transparent red lake paint layer and a more opaque (highly scattering) paint layer can be seen [16]. Vertical dark bands in the cross-section image correspond to the underdrawings which have a high absorption coefficient at $930 \mathrm{~nm}$. The lower half of the cross-section image is dominated by multiply scattered light with scattering centres located in the upper half of the image. This is the result of OCT registering only the optical path length rather than the true physical depth. OCT has been used to detect areas of paint loss hidden beneath the overpaint showing not only the damaged area but also the location of the loss [17]. These areas of loss are not detected in X-ray images. 


\subsection{Underdrawing}

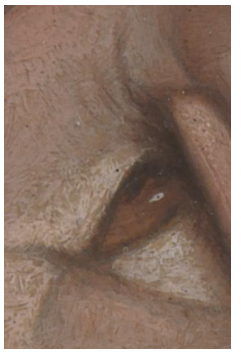

(a)

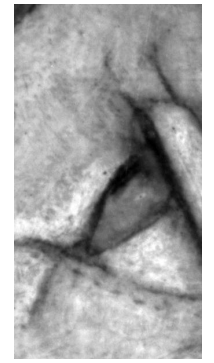

(b)

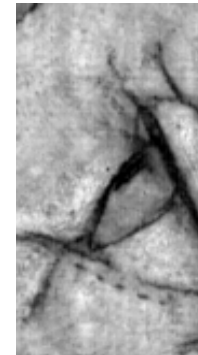

(c)

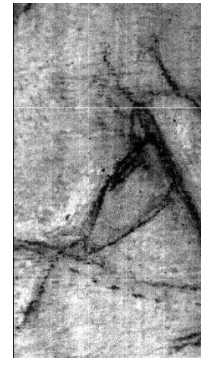

(d)

Fig. 2. a) colour image b) $880 \mathrm{~nm}$ CCD image c) 900-1700nm NIR InGaAS image d) 930nm OCT image (median of 40 en face images) of the eye of the angel in a $15^{\text {th }} \mathrm{C}$ painting attributed to Francesco Francia (National Gallery collection NG3937)

As shown in Fig. 1, OCT can be used to image the underdrawing of a painting through scanning a region and taking en face slices. The best images are obtained through taking the median of a series of en face images at various depths $[5,8,9,12]$. Figure 2 gives a comparison of near infrared images of part of a painting using a CCD camera through a bandpass filter at $880 \mathrm{~nm}$, a state of the art InGaAs digital infrared camera sensitive to $900-1700 \mathrm{~nm}$ and a $930 \mathrm{~nm}$ OCT image (a median image of a series of 40 en face slices in depth). The OCT image gives the highest resolution and dynamic range showing that the underdrawing was drawn with a solid substance. In addition, OCT imaging of underdrawing has the advantage of depth selection, i.e. it can locate the depth position of the underdrawings and median/average only those layers that contain the underdrawing information. Taking the median has the advantage of eliminating the ghost image in any Fourier domain OCT. Speckle noise is also greatly reduced through taking the median or averaging a series of images at different depth. Figure 1 shows that as a result of multiple scattering in the lower paint layers, the shadows of the underdrawing extend far beyond the actual depth location of the underdrawing or the surrounding paint layer. The en face slices below the location of the underdrawing can have higher contrast than at the position of the underdrawing because of the multiply scattered light from the paint layer above the underdrawing. Large area scans of $10 \mathrm{~cm} \times 10 \mathrm{~cm}$ have been achieved through mosaicing of adjacent en face images.

\subsection{Monitoring the cleaning of varnish}

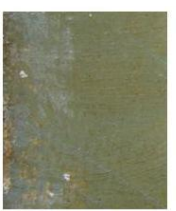

(a)

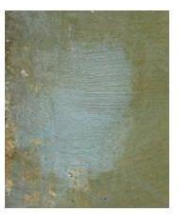

(d)

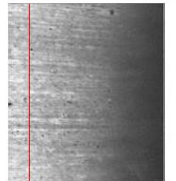

(b)

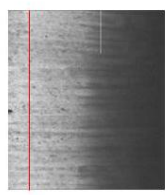

(e)
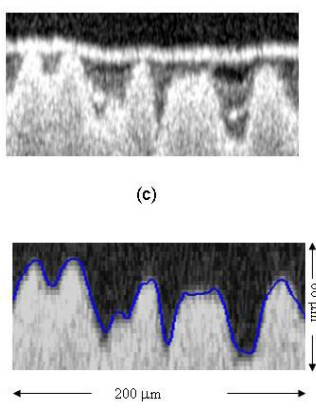

Fig. 3. OCT monitoring of solvent cleaning of varnish: a) part of a painting before cleaning; b) en face OCT image slice at the paint surface below the varnish; c) OCT cross-section image at the position marked in red on the image in b); d) same as a) but after cleaning with industrial methylated spirit/white spirit; e) same as b) but after cleaning; f) same as c) but after cleaning, the blue curve is the paint surface before cleaning obtained from c).

Varnishes have long been applied to traditional Western European paintings for both protective and aesthetic purposes. However, varnishes (especially natural varnishes) degrade over time and become yellow and hazy. It is a routine practice for conservators to remove old varnish and replace it with a coat of new varnish using a solvent that dissolves the old varnish but not the paint. It has not been possible in the past to directly prove that the paint surface is not damaged by the cleaning process. OCT has the potential to directly investigate this as shown in Fig. 3. Figure 3c shows the three varnish 
layers and the paint layer before cleaning. The surface profile of the paint layer before cleaning is obtained after correcting for the optical path length through the varnish above the paint. This paint surface is plotted in blue over the cross-section image at the same position after cleaning. In this case, the difference between the paint surface before and after cleaning is within the measurement error margin.

OCT was first applied to the in situ monitoring of laser cleaning of test paintings by Gora et al. [10]. The full advantage of the fast imaging capability of a Fourier domain OCT was demonstrated in the monitoring of laser cleaning which requires a quick response.

\subsection{Monitoring canvas deformation and vibration}

Canvas deformation as a result of changes in environment such as humidity was first demonstrated using a Fourier domain OCT by Targowski et al. [11]. It was demonstrated that OCT was able to continuously monitor the deformation of the canvas in 3D similar to electronic speckle pattern interferometry. Vibration of canvas paintings as a result of air current from air conditioning ducts and human voice was measured by the Thorlabs SROCT in a conservation studio [9]. In the worst case a peak to peak vibration of $\sim 20 \mu \mathrm{m}$ was found.

\subsection{Measurement of optical properties of paint}

Refractive indices of varnish and paint samples have been measured using OCT $[8,9]$ by either directly measuring the physical thickness and the corresponding optical thickness or using the focus tracking method which has the advantage that it can be used in situ. However, the measurement errors for both methods are large (accuracy of 0.01 to 0.09 ) because of the rather thin layers (tens of microns) and the opacity in the case of paint samples. The methods are limited to transparent layers and can not be applied to paint sample that are highly scattering.

The scattering and absorption coefficients of paint samples can be measured from OCT depth scans and calibrated with the coefficients measured from the Kubelka-Munk theory [22]. Details of this work will be presented in a forth coming publication.

OCT measurements of the scattering and absorption properties can also assist the spectral identification of pigments by providing additional constraints [23].

\section{OCT IMAGING OF OTHER MUSEUM OBJECTS}

\subsection{Glass}

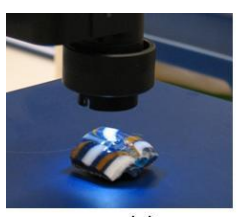

(a)

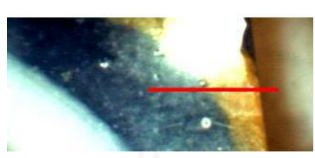

(c)

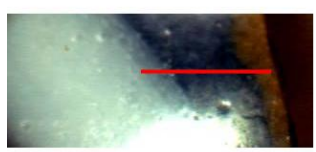

(e)

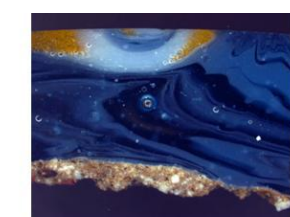

(b)

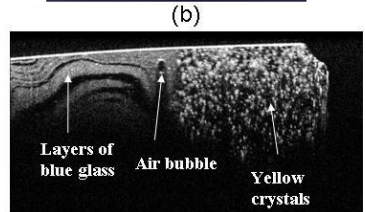

(d)

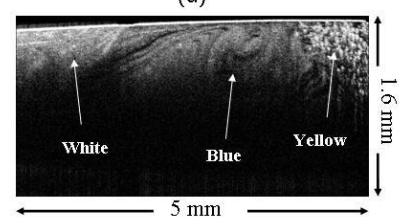

(f)

Fig. 4 Egyptian core formed glass from the British Museum ( 1500 BC) a) OCT probe scanning the glass fragment; b) microscope image of the cross-section of the glass fragment; d) OCT B-scan image of the region marked by the red line segment in c); f) OCT B-scan image of the region marked by the red line segment in e).

OCT has been applied to the subsurface examination of the deterioration of glass [9] and the examination of the internal structure of stained glass [14]. Figure 4 shows the OCT B-scan image of a fragment of Egyptian glass from the British 
Museum compared with a conventional microscope image of the cross-section in a similar region. The blue, yellowish and white areas have different optical properties and structure. The scattering particles are much smaller in the blue and white regions than those in the brown regions. Flow lines and air bubbles are seen in the blue glass. The opacifier crystals in the brown area are well resolved in the OCT image. Opacifiers are normally added to make the glass less transparent and more scattering, typically calcium antimonate crystals are found in white opaque glass and lead antimonate crystals are found in yellow opaque glass [18]. The OCT cross-section image shows that the white layers and the brown layers were applied after the blue layer and that the downward turning of the flow line near the boundaries between the white and blue regions is likely to be the result of the white glass being applied. The ancient manufacturing process of such glass is still a topic for debate. It is thought that the glass was either trailed onto the core by rotating it or the core was dipped into molten glass. The white and yellow decorations were probably added later to the still soft blue glass. A 3D OCT scan gives a $1 \mathrm{~cm} \times 1 \mathrm{~cm} \times 1.6 \mathrm{~mm}$ volume cube that shows that the blue glass consists of different curved layers with very different scattering properties throughout the imaged volume. The best way to test the two hypotheses is to use OCT to image glass samples made by the different proposed methods.

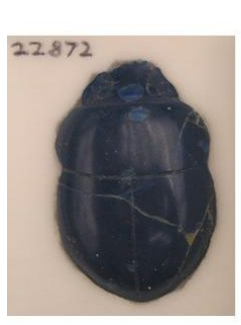

(a)

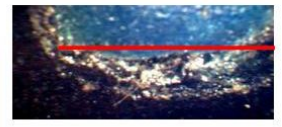

(b)

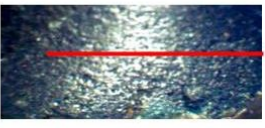

(d)

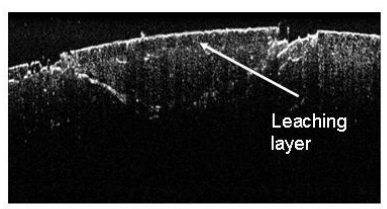

(c)

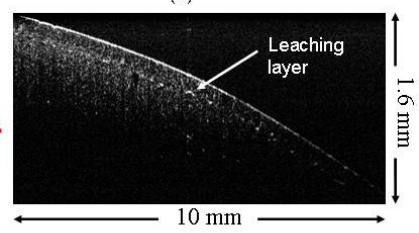

(e)

Fig. 5 An Egyptian glass scarab from the British Musuem (a) showing effect of corrosion in OCT images c) \& d)

Figure 5 shows how OCT can be used to detect glass corrosion non-invasively. The Egyptian scarab has an unstable glass composition which leads to corrosion. Fragments have already fallen off and had to be repaired. The OCT image in Fig. 5c shows how well the glued-in piece fits in the body of the scarab. Figure 5c and 5e show that both the glued-in piece and the original show a leaching layer that extends $\sim 170$ micron in optical thickness below the surface (or $\sim 110$ microns assuming a refractive index of 1.5). The layer has presumably lost its sodium ions and the corrosion starts. OCT can potentially give early warning to glass corrosion.

\subsection{Jade}

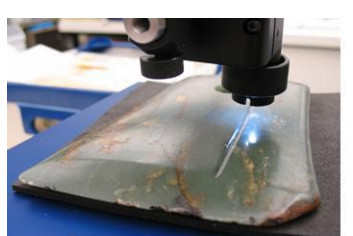

(a)

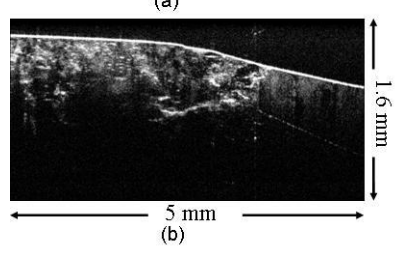

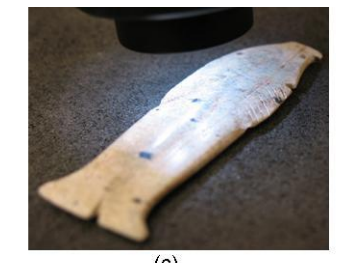

(c)

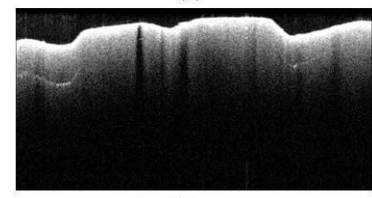

(d)

Fig. 6. a) A Nephrite Jade blade from the British Museum (Neolithic); b) OCT B-scan showing the crystal structure; c) A weathered jade fish from the British Museum; d) B-scan showing strong scattering. 
Figure 6 shows that jade is translucent at 930nm and OCT can image the mineral structure of jade, however, weathered jade is highly scattering with much lower penetration depth at $930 \mathrm{~nm}$. OCT imaging can potentially help to identify the type of jade through their mineral structure. Yang et al. (2004) studied the weathering patterns on jade using OCT in an attempt to distinguish natural weathering from artificial weathering. Figure $6 \mathrm{~d}$ shows some strongly absorbing particles (dark particles seen in Fig. 6c) embedded in the surrounding highly scattering material.

\subsection{Ceramics}
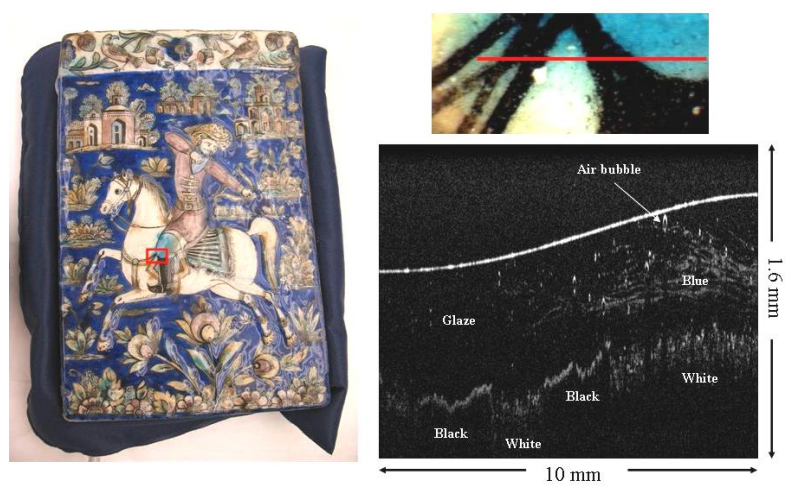

Fig. 7. A 19th century Persian under glaze painted ceramic tile from the British Museum collection.

Micro-XRF (X-ray fluorescence) is used to study the elemental composition of the glaze of the ceramic tile in Fig.7, however, it is difficult without measurements of the thickness of the glaze to know whether the X-rays detected were due to the glaze or the paint layer further down. The OCT image in Fig. 7 shows that the glaze layer is $\sim 800 \mu \mathrm{m}$ in optical thickness which translates to a physical thickness of 400 to $570 \mu \mathrm{m}$ for a refractive index in the range of 1.4 to 2.0 (range of refractive indices found for glass). Theoretical values of the information depth for XRF measurements in an alkaline glass were estimated for a signal fraction of $95 \%$ for various X-ray energy levels. The energy levels for Ca-K alpha line, the Fe-K alpha line and $\mathrm{Pb}-\mathrm{L}$ alpha line are $3.7 \mathrm{keV}, 6.4 \mathrm{keV}$, and $10.5 \mathrm{keV}$ respectively and X-rays at these energy levels can penetrate a depth of about $\sim 20 \mu \mathrm{m}, \sim 60 \mu \mathrm{m}$ and $\sim 200 \mu \mathrm{m}$ respectively [19]. Therefore, the quantification of these main components of the glaze seems to be possible as long as elements with higher energy X-rays are only present as traces. However, the OCT image also shows that most of the blue pigments have dissolved into the glaze, hence XRF measurements of the glaze in this blue area would be contaminated by the blue pigment.

\subsection{Painted enamel}

Painted enamels are made from layers of glass. As in the case of the ceramic tile, XRF is commonly used to identify the material non-invasively. However, an independent method of imaging the layer structure and thickness is needed to associate the elements detected with the correct layer. The OCT images in Fig. 8 show the various layers of glass the enamel is made from. The blue, green and brown layers are transparent, but the white layer is highly scattering and the dark drawings are highly absorbing. In many cases, the layers are transparent enough for the copper plate to be seen in the OCT images. Air bubbles seem to concentrate toward the top surface. The total optical thickness of the enamel layer is $\sim 800 \mu \mathrm{m}$. 


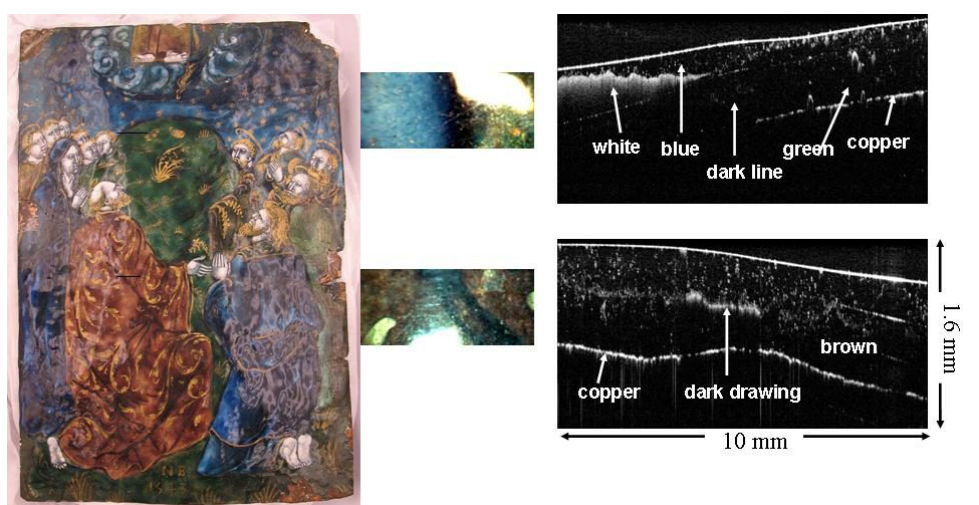

Fig. 8. Painted enamel from the British Museum collection.

\section{REQUIREMENTS FOR OCT IN MUSEUM APPLICATIONS}

While speed is the focus for OCT development in the biomedical field for in vivo imaging, in the field of archaeology and art conservation speed is less of an issue as the objects are stationary. The only applications that require high speed are laser cleaning, tracking of canvas deformation and vibration monitoring. Large area scans can also benefit from higher speed of acquisition even though high speed is not essential for image quality. Most applications in this field do not require high speed. The trade-off between speed and signal to noise ratio is different here. Increase of integration time does not improve the $\mathrm{S} / \mathrm{N}$ of strong OCT signals as these are speckle noise limited. However, for weak signals significant improvements can be made by increasing the integration time. Figure 8 shows the improvement in signal to noise ratio for weak signals in a depth scan through a Ti white paint sample using the 930nm Fourier domain OCT.

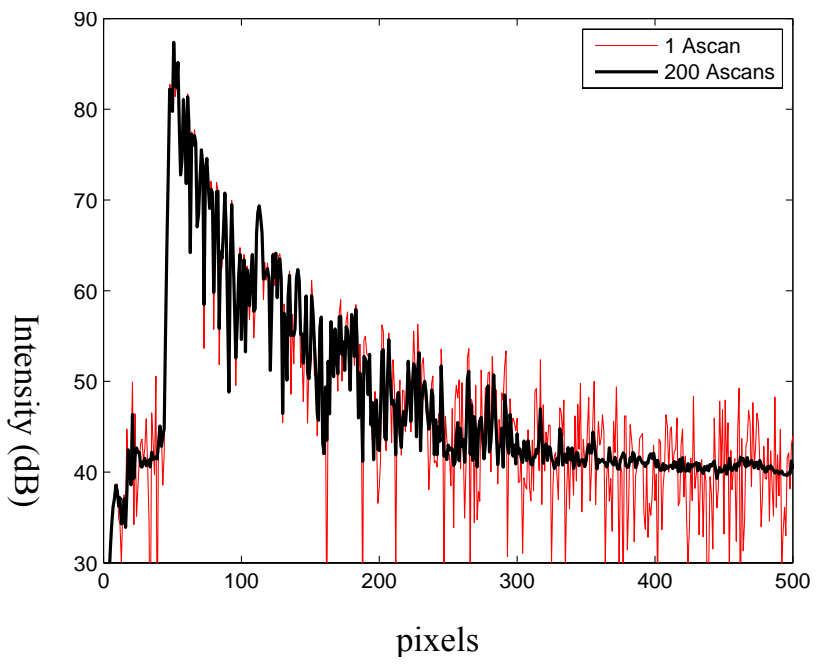

Fig. 9. A depth scan (A-scan) through a Ti white oil paint sample showing the improved signal to noise ratio for an average of 200 A-scans (black) compared with one A-scan (red).

Fourier domain OCT has the advantage of high speed capturing however it has limited depth range which makes imaging of relatively transparent and thick objects difficult. For the Thorlabs SROCT the depth range is $1.6 \mathrm{~mm}$, the relative distance from zero path length gives a $10 \mathrm{~dB}$ drop in signal over $0.9 \mathrm{~mm}$ and the relative distance from the focus position gives another $20 \mathrm{~dB}$ drop in signal over $0.9 \mathrm{~mm}$.

We have designed and built a portable time-domain system for use in situ at the British Museum and National Gallery. The system is illuminated by an $850 \mathrm{~nm}$ centred broadband source (super-luminescent diode) with a FWHM of $50 \mathrm{~nm}$, yielding an axial resolution of approximately 6.7 microns (theoretical) and 8 microns (observed). Depth scanning (scanning of the reference arm) is achieved using mirrors mounted on a translation stage, while lateral scanning is by a 
pair of galvo-mirrors ( $\mathrm{x}$ and $\mathrm{y}$ ). For en-face (transversal) imaging, modulation of the carrier wave is provided by the $\mathrm{x}$ (fast) scanner, no additional phase modulator is required [20]. The system employs balanced detection to reduce excess photon noise [21] and a log scaling of the demodulated signal prior to digitisation in order to preserve dynamic range. The system is enclosed and designed to be easily transportable to imaging sites.

Although the time-domain modality A-scan rate is slower than for Fourier domain OCT, this system has the advantage than it can generate en-face slices at $1 \mathrm{~Hz}$. This finds significant utility in the alignment phase of imaging, since the investigator is presented with a live image which is oriented in the same way as for conventional microscopy (or indeed naked eye viewing), permitting a more intuitive interpretation of structure. The advantage is strongest when investigating features which lie in a plane orthogonal to the optical axis (such as underdrawings) and which may be more difficult to identify in B-scans.

Time-domain OCT also offers the opportunity to acquire single shot images of thick, transparent objects, subject to the limitations of the confocal window (or depth of focus) - the $4 \mathrm{~cm}$ focal length lens normally used provides a (theoretical) depth of focus of $5 \mathrm{~cm}$ in air, for a lateral resolution of 15 microns. This range could, in principle, be extended using dynamic focus, although this has not been implemented at the current time.

\section{CONCLUSIONS}

OCT has great potential to become a routine non-invasive tool in museums for the examination of subsurface structure of painting and other museum objects. As OCT imaging is non-invasive, this means cross-section imaging is possible anywhere on a painting or an intact object where there are no other methods of obtaining the depth information. OCT can go beyond qualitative imaging toward quantitative measurements of optical properties for monitoring the ageing of material and assist material identification.

We would like to thank Kafing Keita of Nottingham Trent University for providing Figure 8, Rachel Billinge of the National Gallery for providing Figure 2c, Margaret Sax and Caroline Cartwright of the British Museum for helpful discussion and David Saunders for support and encouragement.

\section{REFERENCES}

[1] J. Padfield, D. Saunders, J. Cupitt, R. Atkinson, "Improvements in the acquisition and processing of X-ray images of paintings," The National Gallery Technical Bulletin 23, 62-75 (2002).

[2] M.-L. Yang, C.-W. Lu, I.-J. Hsu, C. C. Yang, "The use of optical coherence tomography for monitoring the subsurface morphologies of archaic jades", Archaeometry, 46(2), 171-182 (2004).

[3] P. Targowski, B. Rouba, M. Wojtkowski, and A. Kowalczyk, "The application of optical coherence tomography to non-destructive examination of museum objects", Studies in Conservation, 49(2), 107-114 (2004).

[4] H. Liang, M. Gomez Cid, R. Cucu, G. Dobre, D. Jackson, C. Pannell, J. Pedro, D. Saunders, A. Podoleanu, "Application of OCT to examination of easel paintings", Second European Workshop on Optical Fibre Sensors, Proc. SPIE, 5502, 378-381, (2004).

[5] H. Liang, M. G. Cid, R. G. Cucu, G. M. Dobre, A. Gh. Podoleanu, J. Pedro, D. Saunders, "En-face Optical Coherence Tomography - a novel application of non-invasive imaging to art conservation", Opt. Express, 13, 61336144, (2005). http://www.opticsexpress.org/abstract.cfm?id=85276.

[6] T. Arecchi, M. Bellini, C. Corsi, R. Fontana, M. Materazzi, L. Pezzati, and A. Tortora, "Optical coherence tomography for painting diagnostics", Proc.SPIE 5857, 278-282 (2005).

[7] A. Szkulmowska, M. Góra, M. Targowska, B. Rouba, D. Stifter, E. Breuer, and P. Targowski, "The Applicability of Optical Coherence Tomography at 1.55 um to the Examination of Oil Paintings", in Lasers in the Conservation of Artworks, LACONA VI Proceedings, Vienna, Austria, Sept. 21 - 25, 2005, J. Nimmrichter, W. Kautek, and M. Schreiner, eds. (Springer Verlag, Berlin-Heidelberg-New York, 2007), pp. 487-492

${ }^{[8]}$ H. Liang, M. G. Cid, R. G. Cucu, G. M. Dobre, B. Kudimov, J. Pedro, D. Saunders, J. Cupitt, A. Gh. Podoleanu, "Optical Coherence Tomography - a non-invasive technique applied to conservation of paintings", Proceedings of SPIE, 5857, 261-269 (2005). 
H. Liang, B. Peric, M. Hughes, A. Podoleanu, M. Spring, and D. Saunders, "Optical coherence tomography for art conservation and archaeology", Proc. SPIE 6618, 661805 (2007)

[10] M. Góra, P. Targowski, A. Rycyk, J. Marczak "Varnish ablation control by Optical Coherence Tomography”, Laser Chemistry, Article ID 10647, (2006)

[11] P. Targowski, M. Gora, T. Bajraszewski et al., "Optical coherence tomography for tracking canvas deformation", Laser Chemistry, Article ID 93658, 2006

[12] D. C. Adler, J. Stenger, I. Gorczynska, H. Lie, T. Hensick, R. Spronk, S. Wolohojian, N. Khandekar, J. Y. Jiang, and S. Barry, "Comparison of three-dimensional optical coherence tomography and high resolution photography for art conservation studies", Opt. Express, 15, 15972-15986 (2007).

[13] M. Góra, M. Pircher, E. Götzinger, T. Bajraszewski, M. Strlic, J. Kolar, Ch.K. Hitzenberger, P. Targowski "Optical Coherence Tomography for Examination of Parchment Degradation", Laser Chemistry, Article ID 68679, (2006)

[14] P. Targowski, B. Rouba, M. Góra, L. Tymińska-Widmer, J. Marczak, and A. Kowalczyk, "Optical Coherence Tomography in Art Diagnostics and Restoration” Applied Physics A, 92, 1-9 (2008).

[15] P. Targowski, M. Góra, M. Wojtkowski, "Optical Coherence Tomography for Artwork Diagnostics" , Laser Chemistry, Article ID 35373, (2006) http://www.hindawi.com/GetArticle.aspx?doi=10.1155/2006/35373

[16] H. Liang, B. Peric, M. Spring, D. Saunders, M. Hughes, A. Podoleanu, "A non-invasive imaging of subsurface paint layers with optical coherence tomography", Conservation Science 2007, 171-176, Archtype publishing.

[17] M. Spring, H. Liang, B. Peric, D. Saunders, A. Podoleanu, "Optical Coherence Tomography - a tool for high resolution non-invasive 3D-imaging of the subsurface structure of paintings", Proceeding of ICOM-CC Triennial Conference, 2008, Delhi, Vol. II, 916-923

[18] P. T. Nicholson and J. Anderson, in 'Ancient Egyptian Materials and Technology', P.T. Nicholson \& I. Shaw eds. Cambridge University Press (2000)

[19] Mantler, M., Beckhoff, B. private communication

[20] Podoleanu, A. Gh., Dobre, G.M., Jackson, D.A., "En-face Coherence Imaging Using Galvanometer Scanner Modulation," Opt. Letters 23, 147-149 (1998).

[21] Podoleanu, A. Gh., "Unbalanced versus balanced operation in an optical coherence tomography system," Applied Optics 39(1), 173-182 (2000).

[22] P. Kubelka, "New contributions to the optics of intensely light scattering materials. Part I", J. Opt. Soc. Am. 38, 448-457 (1948).

[23] H. Liang, K. Keita, B. Peric, T. Vajzovic, "Pigment identification with optical coherence tomography and multispectral imaging", The $2^{\text {nd }}$ international topical meeting on optical sensing and artificial vision (OSAV08), May 2008, St Petersburg, in print. 